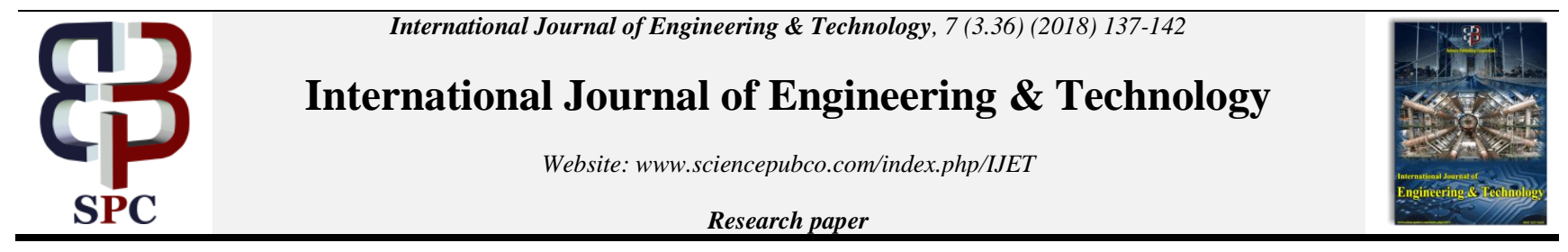

\title{
Form-Finding of Tensegrity Model with Triangular Cells
}

\author{
Nur Farizah Filzah Naing ${ }^{1}$, Oh Chai Lian ${ }^{1 *}$, Ilyani Akmar Abu Bakar'1, Mohd Raizamzamani Md Zain ${ }^{1}$ \\ ${ }^{1}$ Faculty of Civil Engineering, Universiti Teknologi MARA, 40450, Shah Alam, Selangor, Malaysia \\ *Corresponding author E-mail: chailian@salam.uitm.edu.my
}

\begin{abstract}
Tensegrity structures is a light-weight structure compared to concrete structures that are heavy and rigid in shape. The studies on formfinding for tensegrity configuration are still ongoing and have been extensively conducted. Additionally, many proposed tensegrity structures have not been built for real applications. This study aims to determine potential self-equilibrated configurations of three-stage Class I tensegrity model assemblage with triangular cells, which may be applied as deployable towers. The form-finding methodology involves phases in establishment of desired form and formulation for the self-equilibrated state. The system of equilibrium equations was solved by Moore-Penrose generalized inverse method. A range of twist angles $10^{\circ}-50^{\circ}$ for triangular cells was investigated in the form-finding process. It was found that the form-finding method via changing of twist angles has successfully search self-equilibrated tensegrity models.
\end{abstract}

Keywords: Tensegrity; form-finding; cables; stuts; self-equilibrium.

\section{Introduction}

Tensegrity originates from a combination of words tension and integrity. The structure was popularized by Buckminster Fuller since early 1950. It can be seen as a special type of truss which consists of set of continuous tensile members (i.e. cables) and discontinuous compressive members (i.e. struts or bars). The cables act as strings that are attached at the end of axially loaded struts and thus allow no bending moment in the structure. Fuller has described tensegrity as "islands of compressions in an ocean of tension". A three dimensional tensegrity structure based on plywood and monofil sculpture named as $\mathrm{X}$ piece was made by Kenneth Snelson in winter of 1948.

Tensegrity structures are categorized into two broad structural classes that are pre-stressed and geodesic structures. Anthony Pugh was the first person who described the simplest configuration of tensegrity in two-dimensional and three-dimensional by referring to the position of its cables [1]. Pugh have also classified three basic patterns of tensegrity by using the configuration of spherical and cylindrical structures: prism tensegrity, diamond tensegrity and zig-zag tensegrity [2].

The concept of Tensegrity is suitable for the design of deployable structures for space and structural applications. This is owing to the separation of compressive elements in tensegrity structures which makes the folding process easier. Tensegrity mast is a tower-like, prism or cylindrical-like structure with its struts and cables widely extended. Other potential applications of tensegrity structures were suggested such as cable domes, weaving tower structure and Snelson's triangle tensegrity structure [3].

Form-finding is considered as an important step in designing a tensegrity structure. Form-finding can be defined as a process of knowing special geometrical and equilibrium configuration that lead to a state of self-stress for tensegrity structure. There are many methods that have been classified and reviewed for the study of form-finding, particularly kinematical methods and statical method [4]. Statical methods can solve equilibrium equations directly. Several methods that are categorized as statical methods are analytical method, force density method, energy method and reduced coordinates method. Meanwhile, analytical approach, non-linear programming method (i.e. optimization approach) and dynamic relaxation method are considered under kinematical method. Optimization approach is always used in the formfinding strategies. In this approach, the length of cables is kept to constant while the strut length is increased until it reached the maximum length or the strut length is kept constant while the length of cables is decreased until it reached the minimum length [5]. Numerical technique incorporated genetic algorithm with only input of number of nodes has been proposed in solving selfequilibrium state in the form-finding of an irregular tensegrity structure [6]. Feasible solution for nodal coordinates and force densities has been determine by performing singular value decomposition of equilibrium matrix has also been investigated [7]. Form-finding of tensegrity mast $[8,9]$ and tensegrity mimicking human backbone [10] have been carried out.

In this study, form-finding of three-stage (Class I) tensegrity models with triangular cell was carried out by using computational method by [8]. Other than that, investigation of axial forces for the three-stage tensegrity models at self-equilibrated state was also presented. The authors hope that this study could further contributes to the field of form-finding of tensegrity structure.

The remainder of the paper is organized as follows. Section 2 presents the methodology of form-finding for multiple-stage tensegrity model. Section 3 shows the numerical examples for three-stage tensegrity model $\mathrm{T} 1$ and $\mathrm{T} 2$ using different triangular cells. Section 4 presents the axial forces of the tensegrity models at self-equilibrium state. Finally, Section 5 shows the concluding remarks for the paper.

\section{Form-finding Procedure}

This section presents the procedure of form-finding for three-stage (Class I) tensegrity model with triangular cells. The procedure 
includes the establishment of element connectivity, preparation of geometrical inputs, and determination of self-equilibrium stress state and analysis of slackened cables. Form-finding for threestage tensegrity models was carried out by using proposed computational method by Oh et al. (2016). Summary of the methodology is shown in Figure 1.

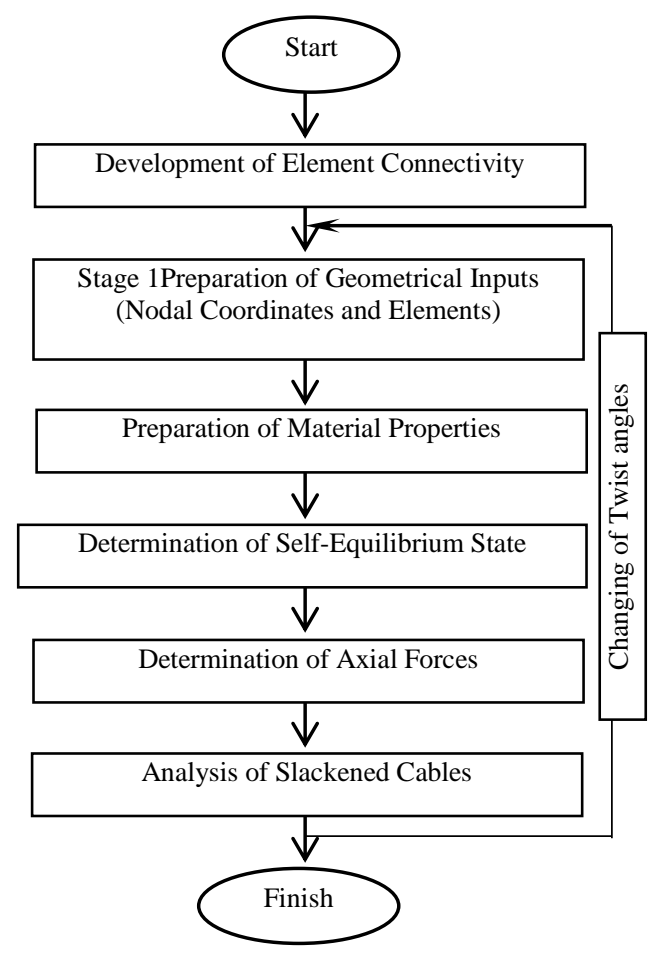

Fig. 1: Form-finding Procedure

\subsection{Terminology}

Several terminologies have been used in this study such as tensegrity mast, elements and nodes, stage, sag depth, twist angle and vertex.

- Tensegrity mast is defined as a tower like structure that are prism and cylindrical shape between the struts and cables. Figure 2 shows an example of tensegrity mast that consists of three struts and nine cables.

- Elements refer to struts and cables whereas nodes refer to the joints between struts and cables.

- Stage is defined as the numbers of triangular cells when the cells are stacking up to form a tensegrity mast. A tensegrity mast can be assembled from stage 1,2 and up to stage $n$. In this study, tensegrity mast consists of three triangular cells, which the cells are stacked up until stage $3(n=3)$.

- Sag depth is defined as a height between the top nodes of cells at lower stage (i.e. stage 1) and lower nodes of cells at upper stage (i.e. stage 2). It is also known as saddle height.

- Twist angle is a difference is rotational angle of the upper triangular surface of a cell with respect to the lower triangular surface. The vertex angle is the interior angle of the triangle for the triangular surfaces.

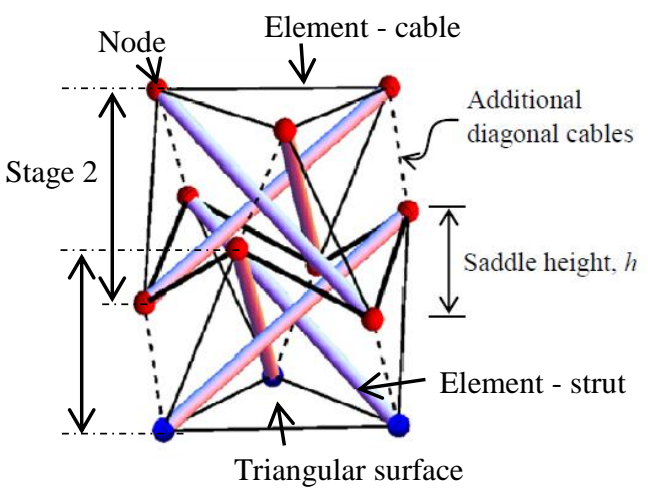

Fig. 2: Example of tensegrity mast

\subsection{Three-stage tensegrity model}

A total of two three-stage tensegrity models with triangular cell, namely models $\mathrm{T} 1$ and $\mathrm{T} 2$ are investigated in the study. Triangular cell is chosen for the models because of its simplicity in geometry. A triangular cell consists of nine cables and three struts. The cables have been divided into two parts, mainly the horizontal and diagonal cables. The horizontal cables are located horizontally at the bottom and top of the model whereas the diagonal cables connected struts between cells.

The form-finding process starts with the development of element connectivity followed by preparation of geometrical input such as nodal coordinate. Nodal coordinates of the tensegrity model are firstly identified in order to assemble the model by using triangular surface as shown in Figure 3. Table 1 shows the difference vertex angles in model T1 and T2. In a triangular cell, each stage of the tensegrity model consists of six nodes respectively. Therefore, in this study, a total of 18 nodal coordinates are determined to establish a three-stage tensegrity model.

Figure 4 shows the element connectivity chart developed by Oh et al. (2016). It is noted from the chart that the cells are stacked up in stages. $L_{i} B$ denotes the position of lower triangular surface of the first stage cell whereby $L_{i} T$ is positioned at upper triangular surface of cell- $i$ (see Figure 3). $i$ is numbers of stage in the model (i.e. $i=1,2, \ldots, 3)$. In this study, the element connectivity of the three-stage tensegrity models $\mathrm{T} 1$ and $\mathrm{T} 2$ are arranged according to the element connectivity chart in Figure 4.

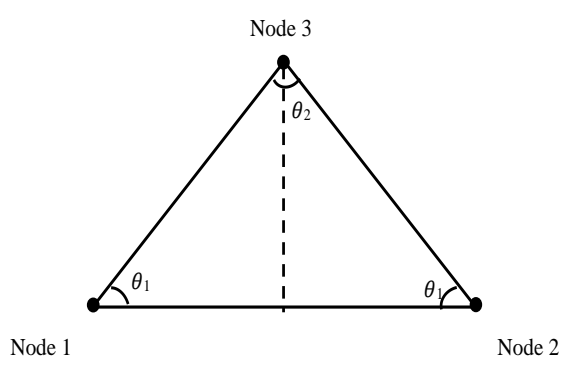

Fig. 3: Plan view triangular surface at base

Table 1: Vertex angle

\begin{tabular}{ccc}
\hline \multirow{2}{*}{ Model } & \multicolumn{2}{c}{ Vertex angle } \\
\cline { 2 - 3 } & $\theta_{1}\left({ }^{\circ}\right)$ & $\theta_{2}\left({ }^{\circ}\right)$ \\
\hline T1 & 55 & 70 \\
\hline T2 & 60 & 60 \\
\hline
\end{tabular}




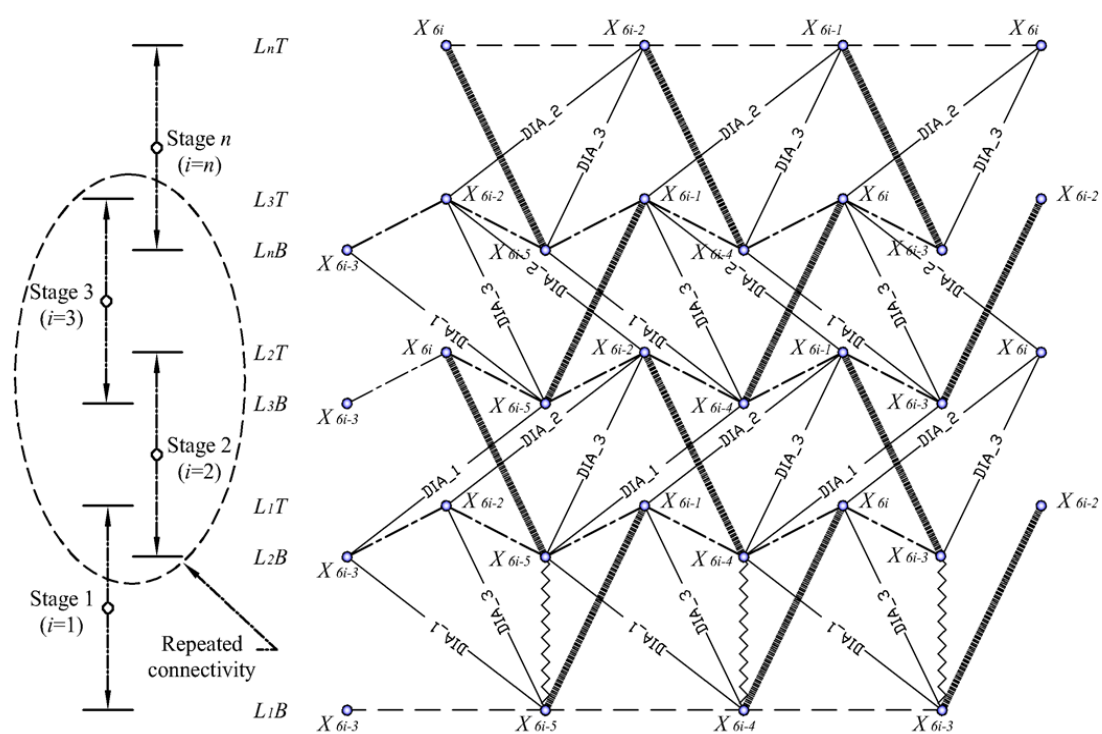

Fig. 3: Element connectivity developed by Oh et al (2016)

Next, material properties for struts and cables are prepared for determining the upper and lower limit of axial forces. The material used for the struts and cables are steel. The value of Young Modulus for the material is $200 \mathrm{GPa}$. Cross sectional area of struts and cables are $50.3 \mathrm{~mm}^{2}$ and $3.14 \mathrm{~mm}^{2}$, respectively. The density of steel is $7.7 \times 10^{-5} \mathrm{~kg} / \mathrm{mm}^{3}$ and yield strength is $250 \mathrm{MPa}$. No selfweight is considered in the study.

Once the topology and material properties of the models are prepared, formulation of self-equilibrium condition of the models are carried out. Basic formulation of equilibrium equation of tensegrity model is same as model with pinned jointed. The nodal coordinates for an element particularly at near end $i$ and far end $j$ in the model are expressed as

$$
\boldsymbol{x}_{i}=\left\{\begin{array}{l}
x_{i} \\
y_{i} \\
z_{i}
\end{array}\right\}, \boldsymbol{x}_{j}=\left\{\begin{array}{l}
x_{j} \\
y_{j} \\
z_{j}
\end{array}\right\}
$$

Thus, total length of element can be determined as

$$
L_{T_{k}}=\sqrt{\left(x_{j}-x_{i}\right)^{T}\left(x_{j}-x_{i}\right)}
$$

The directional cosine of an element is expressed as

$\lambda_{k}=\frac{\left(\boldsymbol{x}_{j}-\boldsymbol{x}_{i}\right)}{L_{T_{k}}}$

Static equilibrium equation is obtained as follows

$$
f=B n
$$

where $\boldsymbol{f}$ is vector of external forces, $\boldsymbol{B}$ is stiffness matrix and $\boldsymbol{n}$ is vector of axial forces. Equation 4 is reduced if the tensegrity model is in the state of self-equilibrium state without external forces, $\boldsymbol{f}=0$. No self-weight is considered in the study and the expression is given as

$$
B \boldsymbol{n}=\mathbf{0}
$$

The vector of axial force $\boldsymbol{n}$ can be solved by the following equation:

$$
\boldsymbol{n}=\left[\boldsymbol{I}_{m}-\boldsymbol{B}^{+} \boldsymbol{B}\right] \boldsymbol{\beta}
$$

where $\beta$ is an arbitrary vector of coefficient of size $m$, unless the trivial solution exists. $m$ is numbers of elements in the model.

Microsoft Office Excel Solver tool is used to search one possible combination of coefficient $\beta$. The coefficient $\beta$ is used to determine axial forces (see Equation 6). In order to solve the variables, conjugate gradient method is utilized. Inequality constraints are imposed in the minimization problem to obtain a self-equilibrated model that compatible with the material of properties. The constraints ensure that the axial forces for cables and struts are kept within their lower and upper bound limit:

$0 \leq n_{c} \leq \sigma_{c} A_{c}$

$-\frac{\pi^{2} E_{s} I_{s}}{l_{s}^{2}} \leq n_{s} \leq \sigma_{s} A_{s}$

where $n_{c}=$ axial forces for cables, $\sigma_{c}=$ yield stress for cables, $A_{c}=$ cross sectional area for cable elements, $n_{s}=$ axial forces for struts, $E_{s}=$ Young Modulus for struts, $I_{s}=$ moment of inertia for struts, $L_{s}$ $=$ element length for struts, $\sigma_{s}=$ yield stress for struts, $A_{s}=$ cross sectional area for strut elements.

It is noted that the cross sectional area for struts in tensegrity model T1 and T2 is circular. Using Euler's buckling load, the compressive forces of strut element are determined.

Additionally, the self-equilibrium stress nodes are extracted for the analysis of slackened cables. When there are none slackened cables in the model, the tensegrity models are said to be in the self-equilibrium condition and the form-finding process stopped. Consequently, the axial forces for tensegrity models in the prestress condition can be determined.

\section{Results and Discussions}

This section presents the results and discussion from the formfinding of three-stage (Class I) tensegrity models, particularly the analysis of slackened cables and self-equilibrated configuration. Each of the section follows the aforementioned results for model $\mathrm{T} 1$ and $\mathrm{T} 2$ and the results discussed.

The configuration of the tensegrity model can be searched based on the form finding results. There are two different vertex angles as shown in Table 1 that had been applied in order to obtain the initial nodal coordinates for the model. A total of 18 nodes and 51 
elements, specifically 9 struts and 42 cables were established for $\mathrm{T} 1$ and $\mathrm{T} 2$. The element connectivity and nodal coordinates are shown in Appendix.

Twist angles are applied on initial topology of the tensegrity model to search for the self-equilibrated configuration through formfinding process. The application of twist angle with range of between 10 - 50 degrees is investigated in the study. Trial configuration are analyzed after the application of twist angles for initial configuration. It is noted that only the $\mathrm{x}$ and $\mathrm{y}$-coordinate of triangular surfaces changed after the application of twist angles. The zcoordinate remain the same as the initial due to constant height. Numbers of slackened cables are studied during the search of the self-equilibrated model. The models are considered to be in the self-equilibrated state when all the cables in tension which means there is none slackened cables.

\subsection{Model T1}

Figure 5 shows the number of slackened cables against the twist angle for model T1. Firstly, analysis cases with application of twist angle $10^{\circ}-50^{\circ}$, with interval of $10^{\circ}$ were studied for model T1. The number of slackened cables decreased significantly for the analysis cases with increasing twist angle. Figure 5 shows that the maximum and minimum number of slackened cables are 20 and 1, respectively when the application of different twist angles. It shows that the lowest number of slackened cables is recorded for twist angle $50^{\circ}$ but the form-finding process has yet to meet the criteria where there should be none slackened cables exists in the tensegrity model.

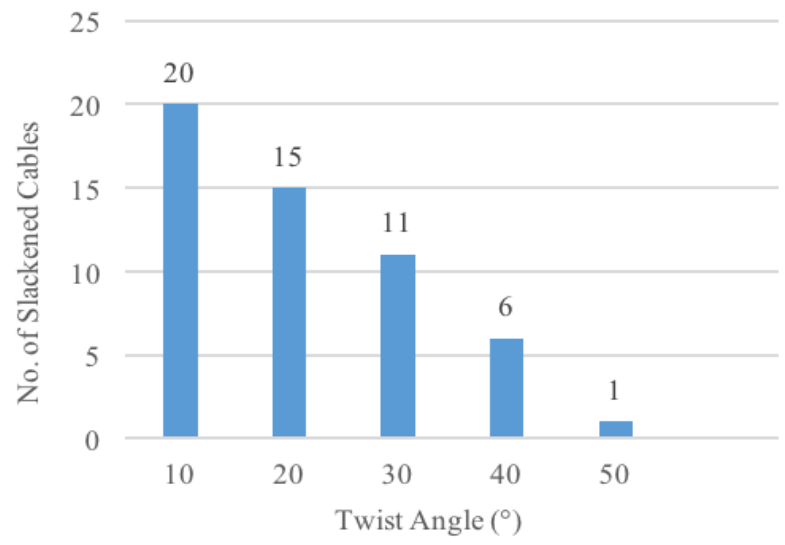

Fig. 5: Number of slackened cables at various $\left(10-50^{\circ}\right)$ twist angles for model T1

Based on Figure 5, it shows that the number of slackened cables between twist angle $40^{\circ}-50^{\circ}$ is decreasing and getting closer to 0 which is none slackened cables. So, the twist angle with range 40$50^{\circ}$ has been investigated in the next phase. Since the number of slackened cables at twist angle $45^{\circ}-50^{\circ}$ is lower compared to slackened cables at $40^{\circ}-45^{\circ}$, only the results are shown in Figure 6. The form-finding continues with the analysis cases with twist angles between $45-50^{\circ}$ and its interval is $1^{\circ}$. From twist angle of $45^{\circ}$ to $46^{\circ}$, there is a significant reduction in number of slackened cables from 4 to 2 . The number of the slackened cables remain constant for both twist angle $46^{\circ}$ and $47^{\circ}$. It is found that there is no slackened cable at twist angle at $48^{\circ}$ which denotes the satisfaction of the form-finding process and the process stop. Figure 7 shows the self-equilibrated model T1.

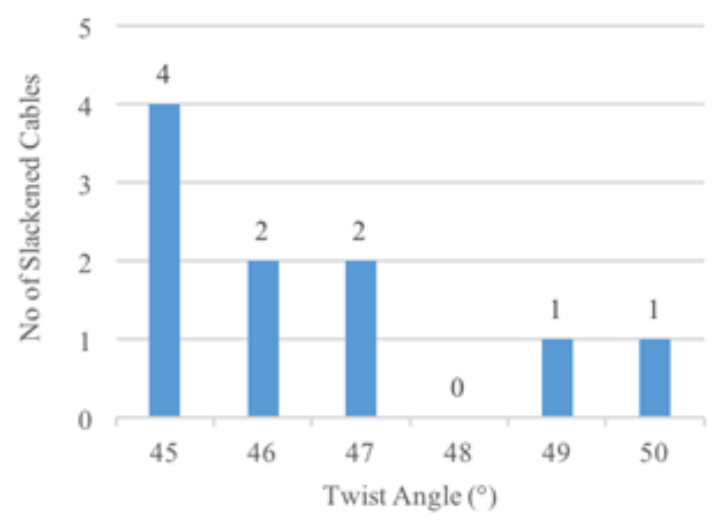

Fig. 6: Number of slackened cables at twist angle $\left(45-50^{\circ}\right)$ for model T1

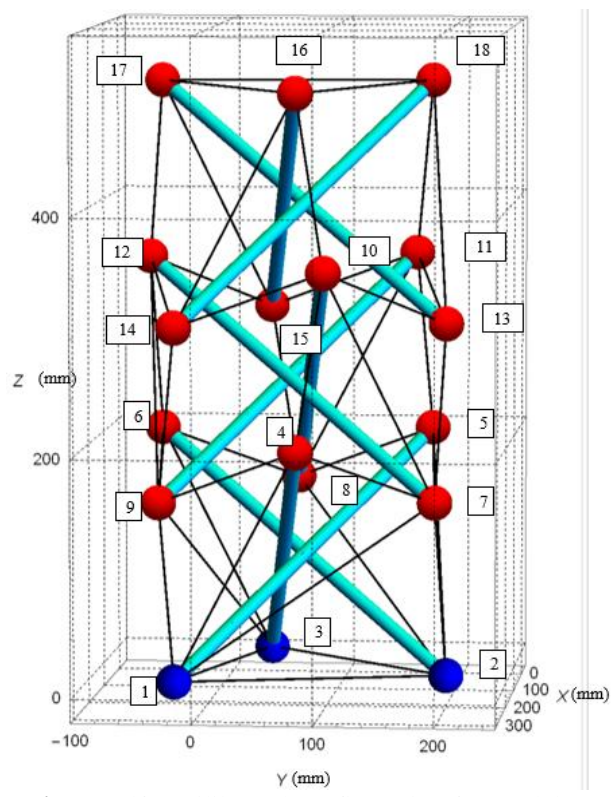

Fig.7: Self-equilibrated configuration for model T1

\subsection{Model T2}

For model T2, Figure 8 shows the graph of number of slackened cables against twist angle. Again, the twist angle of $10^{\circ}-50^{\circ}$ is applied to the model $\mathrm{T} 2$ with $10^{\circ}$ as the interval. The number of slackened cables decreased as the twist angle increased. The maximum number of slackened cables is 17 at application of twist angle $10^{\circ}$ whereby the lowest is 0 at twist angle $50^{\circ}$. This means the configuration of self-equilibrated model $\mathrm{T} 2$ is found at twist angle $50^{\circ}$ as shown in Figure 9.

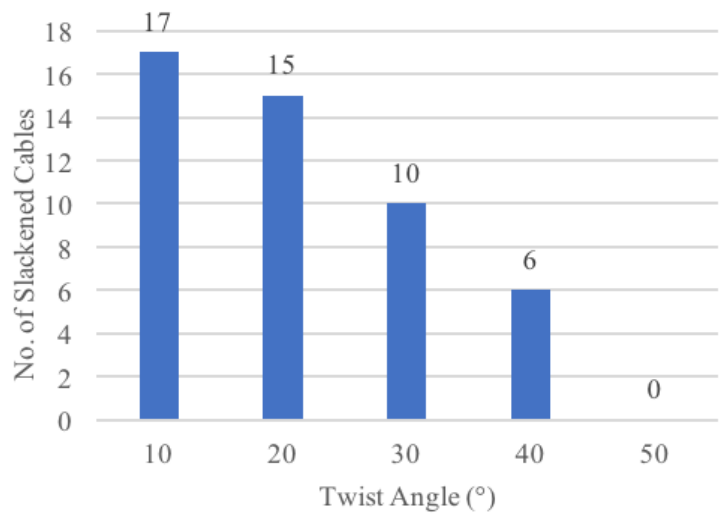

Fig. 8: Number of slackened cables at various twist angle $\left(10^{\circ}-50^{\circ}\right)$ for model T2 
In the real world environment, the elements of the tensegrity model shall not collide to each other. The form-finding analysis that allows the successful search of the self-equilibrated configuration shall incorporate dimensions in the computational strategy. In this study, both model T1 and T2 established with $8 \mathrm{~mm}$ struts do not show any internal collision by observation.

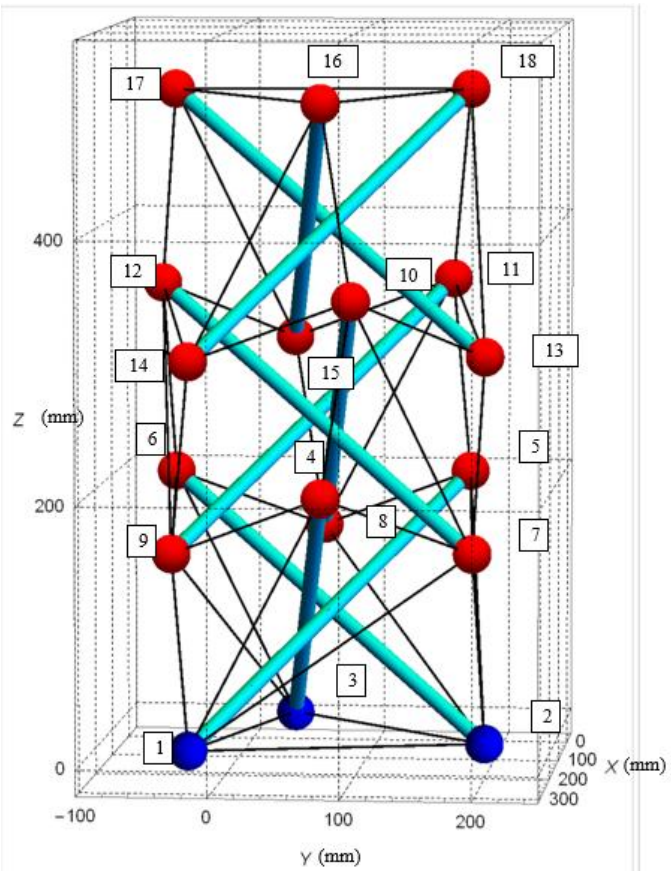

Fig.9: Self-equilibrated configuration for model T2

All of the axial forces for both models are in between the lower and upper bound limit for cables and struts. Axial forces for both models is shown in Appendix. In this study, elements $1-9$ are considered as struts whereby the other elements are the cables. All of the struts obtained negative axial forces which means that the struts are in compression mode. Model T1 shows the highest axial force for struts is $-203.975 \mathrm{~N}$ whereby for model $\mathrm{T} 2$, the value is $271.859 \mathrm{~N}$. Both of the highest compressive forces are observed in strut 7.

Elements 10 to 51 are cables. Numbers of diagonal cable are more than the saddle and horizontal cables. The cables are considered as slackened cable if the axial forces are negative. All of the values should be positive. The highest axial forces of cables for model T1 and T2 are $168.490 \mathrm{~N}$ (at element 47) and $203.374 \mathrm{~N}$ (at element 41 ), respectively. In addition, by comparing both models, the diagonal cables element shows the lowest axial forces among all of the cables.

\section{Conclusions}

In this study, two self-equilibrated configuration of three-stage Class I tensegrity models $\mathrm{T} 1$ and $\mathrm{T} 2$ are successfully search through a form-finding method. Configuration of the models T1 and $\mathrm{T} 2$ are differentiated with the value of vertex angle in the triangular cell. Both the models satisfy the criteria of upper and lower limits of axial force. Both the models are analysed with real elemental sizes has also show internal collision free. It was found that the number of axial forces can be changed easily when there is slight change in the geometry (i.e. change in twist angle and slight difference in vertex angle). The adopted form-finding method is able to find self-equilibrated tensegrity models based on a given initial topology. The axial forces can be determined by after the form-finding analysis with none slackened cable.

\section{Acknowledgement}

The authors would like to thank Ministry of Higher Education Malaysia through FRGS grant (FRGS/1/2017/TK01/UITM/02/1) for funding this study. We would like to express our appreciation to University Teknologi MARA (UiTM), Selangor in providing the facilities.

\section{Appendix}

Table A.1 and Table A. 2 show the nodal coordinates of the initial topology for model T1 and T2, respectively. Table A.3 shows the element connectivity for both models. Table A.4. shows the axial forces of both models.

Table A.1: Nodal coordinates for self-equilibrated model T1

\begin{tabular}{|c|c|c|c|c|c|}
\hline \multirow{2}{*}{ Nodes } & \multicolumn{3}{|c|}{ Coordinates } & \multirow{2}{*}{ Position } & \multirow{2}{*}{$\begin{array}{c}\text { Triangular } \\
\text { Surface }\end{array}$} \\
\hline & $\mathbf{x}$ & $\mathbf{y}$ & $\mathbf{z}$ & & \\
\hline N1 & -12.48 & 38.51 & 0 & L1B & \multirow[t]{3}{*}{ 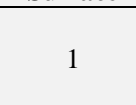 } \\
\hline $\mathrm{N} 2$ & 257.98 & -33.96 & 0 & L1B & \\
\hline N3 & 174.51 & 195.46 & 0 & L1B & \\
\hline $\mathrm{N} 4$ & 58.90 & -65.49 & 200 & L1T & \multirow{3}{*}{2} \\
\hline N5 & 293.72 & 87.00 & 200 & L1T & \\
\hline N6 & 67.38 & 178.49 & 200 & L1T & \\
\hline N7 & 213.90 & -69.65 & 150 & L2B & \multirow{3}{*}{3} \\
\hline $\mathrm{N} 8$ & 199.25 & 209.96 & 150 & L2B & \\
\hline N9 & 6.85 & 59.69 & 150 & L2B & \\
\hline N10 & 88.15 & -79.47 & 350 & L2T & \multirow{3}{*}{4} \\
\hline N11 & 286.14 & 118.52 & 350 & L2T & \\
\hline N12 & 45.72 & 160.95 & 350 & L2T & \\
\hline N13 & -12.48 & 38.51 & 300 & L3B & \multirow{3}{*}{5} \\
\hline N14 & 257.98 & -33.96 & 300 & L3B & \\
\hline N15 & 174.51 & 195.46 & 300 & L3B & \\
\hline N16 & 58.90 & -65.49 & 500 & L3T & \multirow{3}{*}{6} \\
\hline N17 & 293.72 & 87.00 & 500 & L3T & \\
\hline N18 & 67.38 & 178.49 & 500 & L3T & \\
\hline
\end{tabular}

Table A.2: Nodal coordinates for self-equilibrated model T2

\begin{tabular}{|c|c|c|c|c|c|}
\hline \multirow{2}{*}{ Nodes } & \multicolumn{3}{|c|}{ Coordinates } & \multirow{2}{*}{ Position } & \multirow{2}{*}{$\begin{array}{c}\text { Triangular } \\
\text { Surface }\end{array}$} \\
\hline & $\mathbf{x}$ & $\mathbf{y}$ & $\mathbf{z}$ & & \\
\hline N1 & 0 & 0 & 0 & L1B & \multirow{3}{*}{1} \\
\hline N2 & 230.94 & 0 & 0 & L1B & \\
\hline N3 & 115.47 & 200 & 0 & L1B & \\
\hline N4 & 0 & 0 & 200 & L1T & \multirow{3}{*}{2} \\
\hline N5 & 230.94 & 0 & 200 & L1T & \\
\hline N6 & 115.47 & 200 & 200 & L1T & \\
\hline N7 & 0 & 0 & 150 & L2B & \multirow{3}{*}{3} \\
\hline N8 & 230.94 & 0 & 150 & L2B & \\
\hline N9 & 115.47 & 200 & 150 & L2B & \\
\hline N10 & 0 & 0 & 350 & L2T & \multirow{3}{*}{4} \\
\hline N11 & 230.94 & 0 & 350 & L2T & \\
\hline N12 & 115.47 & 200 & 350 & L2T & \\
\hline N13 & 0 & 0 & 300 & L3B & \multirow{3}{*}{5} \\
\hline N14 & 230.94 & 0 & 300 & L3B & \\
\hline N15 & 115.47 & 200 & 300 & L3B & \\
\hline N16 & 0 & 0 & 500 & L3T & \multirow{3}{*}{6} \\
\hline N17 & 230.94 & 0 & 500 & L3T & \\
\hline N18 & 115.47 & 200 & 500 & L3T & \\
\hline
\end{tabular}

Table A.3: Element connectivity of $\mathrm{T} 1$ and $\mathrm{T} 2$

\begin{tabular}{|c|c|c|c|}
\hline Description & Member & Node $\boldsymbol{i}$ & Node $\boldsymbol{j}$ \\
\hline \multirow{3}{*}{ Strut (L1) } & 1 & 1 & 5 \\
\cline { 2 - 4 } & 2 & 2 & 6 \\
\cline { 2 - 4 } & 3 & 3 & 4 \\
\hline \multirow{3}{*}{ Strut (L2) } & 4 & 7 & 12 \\
\cline { 2 - 4 } & 5 & 8 & 10 \\
\hline \multirow{3}{*}{ Strut (L3) } & 6 & 9 & 11 \\
\cline { 2 - 4 } & 7 & 13 & 17 \\
\cline { 2 - 4 } & 8 & 14 & 18 \\
\hline Horizontal cable & 9 & 15 & 16 \\
\cline { 2 - 4 } (base) & 10 & 1 & 3 \\
\hline
\end{tabular}




\begin{tabular}{|c|c|c|c|}
\hline & 12 & 3 & 1 \\
\hline \multirow{3}{*}{$\begin{array}{l}\text { Horizontal cable } \\
\text { (top) }\end{array}$} & 13 & 17 & 18 \\
\hline & 14 & 18 & 16 \\
\hline & 15 & 16 & 17 \\
\hline \multirow{3}{*}{$\begin{array}{l}\text { Additional diagonal } \\
\text { cable (L1) }\end{array}$} & 16 & 1 & 7 \\
\hline & 17 & 2 & 8 \\
\hline & 18 & 3 & 9 \\
\hline \multirow{3}{*}{ Diagonal 1 (L1) } & 19 & 1 & 9 \\
\hline & 20 & 2 & 7 \\
\hline & 21 & 3 & 8 \\
\hline \multirow{3}{*}{ Diagonal 1 (L2) } & 22 & 9 & 13 \\
\hline & 23 & 7 & 14 \\
\hline & 24 & 8 & 15 \\
\hline \multirow{3}{*}{ Diagonal 2 (L2) } & 25 & 4 & 10 \\
\hline & 26 & 5 & 11 \\
\hline & 27 & 6 & 12 \\
\hline \multirow[t]{3}{*}{ Diagonal 2 (L3) } & 28 & 10 & 16 \\
\hline & 29 & 11 & 17 \\
\hline & 30 & 12 & 18 \\
\hline \multirow[t]{3}{*}{ Diagonal 3 (L1) } & 31 & 1 & 4 \\
\hline & 32 & 2 & 5 \\
\hline & 33 & 3 & 6 \\
\hline \multirow[t]{3}{*}{ Diagonal 3 (L2) } & 34 & 7 & 10 \\
\hline & 35 & 8 & 11 \\
\hline & 36 & 9 & 12 \\
\hline \multirow[t]{3}{*}{ Diagonal 3 (L3) } & 37 & 13 & 16 \\
\hline & 38 & 14 & 17 \\
\hline & 39 & 15 & 18 \\
\hline \multirow[t]{6}{*}{ Saddle cable (IM1) } & 40 & 9 & 4 \\
\hline & 41 & 4 & 7 \\
\hline & 42 & 7 & 5 \\
\hline & 43 & 5 & 8 \\
\hline & 44 & 8 & 6 \\
\hline & 45 & 6 & 9 \\
\hline \multirow[t]{6}{*}{ Saddle cable (IM2) } & 46 & 15 & 12 \\
\hline & 47 & 12 & 13 \\
\hline & 48 & 13 & 10 \\
\hline & 49 & 10 & 14 \\
\hline & 50 & 14 & 11 \\
\hline & 51 & 11 & 15 \\
\hline
\end{tabular}

Table A.4: Axial forces of self-equilibrated model T1 and T2

\begin{tabular}{|c|c|c|}
\hline \multirow{2}{*}{ Element } & \multicolumn{2}{|c|}{ Axial Forces (kN) } \\
\hline & T1 & T2 \\
\hline 1 & -131.669 & -201.942 \\
\hline 2 & -123.427 & -199.876 \\
\hline 3 & -194.320 & -253.844 \\
\hline 4 & -176.514 & -244.811 \\
\hline 5 & -193.200 & -270.393 \\
\hline 6 & -188.350 & -258.941 \\
\hline 7 & -203.957 & -271.859 \\
\hline 8 & -203.599 & -268.658 \\
\hline 9 & -148.944 & -248.972 \\
\hline 10 & 26.944 & 94.764 \\
\hline 11 & 73.039 & 113.957 \\
\hline 12 & 78.092 & 97.7102 \\
\hline 13 & 116.804 & 137.149 \\
\hline 14 & 73.413 & 111.386 \\
\hline 15 & 53.982 & 99.303 \\
\hline 16 & 3.276 & 0.00001 \\
\hline 17 & 8.472 & 24.426 \\
\hline 18 & 65.564 & 56.345 \\
\hline 19 & 17.752 & 20.649 \\
\hline 20 & 60.381 & 65.765 \\
\hline 21 & 75.429 & 74.307 \\
\hline 22 & 19.724 & 24.654 \\
\hline 23 & 45.326 & 50.296 \\
\hline 24 & 54.242 & 49.235 \\
\hline 25 & 32.846 & 44.748 \\
\hline 26 & 0.0001 & 10.647 \\
\hline 27 & 18.882 & 40.899 \\
\hline 28 & 59.488 & 77.633 \\
\hline 29 & 32.261 & 35.798 \\
\hline 30 & 85.184 & 101.526 \\
\hline
\end{tabular}

\begin{tabular}{|c|c|c|}
\hline 31 & 61.783 & 64.026 \\
\hline 32 & 11.811 & 37.483 \\
\hline 33 & 0.000 & 10.896 \\
\hline 34 & 55.027 & 76.658 \\
\hline 35 & 60.470 & 81.814 \\
\hline 36 & 57.666 & 61.402 \\
\hline 37 & 32.314 & 50.108 \\
\hline 38 & 93.406 & 87.907 \\
\hline 39 & 37.589 & 70.612 \\
\hline 40 & 144.043 & 198.424 \\
\hline 41 & 135.323 & 203.374 \\
\hline 42 & 106.061 & 170.662 \\
\hline 43 & 105.504 & 186.359 \\
\hline 44 & 133.979 & 188.124 \\
\hline 45 & 122.581 & 155.956 \\
\hline 46 & 163.779 & 191.301 \\
\hline 47 & 168.490 & 200.918 \\
\hline 48 & 144.326 & 178.865 \\
\hline 49 & 146.046 & 179.817 \\
\hline 50 & 131.117 & 159.581 \\
\hline 51 & 139.295 & 165.303 \\
\hline
\end{tabular}

\section{References}

[1] Jáuregui, V. G. Tensegrity structures and their application to architecture: PubliCan, ediciones de la Universidad de Cantabria, (2010).

[2] Pugh, A. An introduction to tensegrity. Univ of California Press. (1976).

[3] Snelson, K. "The art of tensegrity", International journal of space structures, Vol.27, No.2-3, (2012), pp. 71-80.

[4] Tibert, A., \& Pellegrino, S. "Review of form-finding methods for tensegrity structures", International journal of space structures, Vol.18, No.4, (2003), pp.209-223.

[5] Ohsaki, M., \& Zhang, J. "Nonlinear programming approach to form-finding and folding analysis of tensegrity structures using fictitious material properties", International Journal of Solids and Structures, Vol.69, (2015). pp.1-10.

[6] Gan, B. S., Zhang, J., Nguyen, D. \& Nouchi, E. "Node-based Genetic Form-finding of Irregular Tensegrity Structure", Computers and Structures. Vol.159, (2015), pp. 61-73.

[7] Lee, S., Lee, J. \& Kang, J. A. "Genetic Algorithm Based Formfinding of Tensegrity Structures with Multiple Self-stress States", Journal of Asian Architecture and Building Engineering. Vol.162, No.1, (2016), pp. 155-162.

[8] Oh, C. L., Choong, K. K., Nishimura, T., \& Lee, S. W. "Selfequilibrated Tapered Three-stage Tensegrity Mast", Journal of Physics: Conference Series, Vol. 1005, No. 1, (2018), pp. 012039.

[9] Oh, C. L., Choong, K. K., Nishimura, T., Kim, J. Y., \& Zain, M. R. "Tapered three-stage deployable tensegrity model", Journal of Physics: Conference Series, Vol.1130, No. 1, (2018), pp. 012031.

[10] Oh, C. L., Choong, K. K., Nishimura, T. \& Kim, J.-Y. "Formfinding of Human Spine Inspired Biotensegrity", Proceedings of the IASS Annual Symposium 2016, 2016 Tokyo. 